\title{
Mid-Infrared Trace Gas Sensor Technology Based on Intracavity Quartz-Enhanced Photoacoustic Spectroscopy
}

\author{
Jacek Wojtas ${ }^{1, *}$, Aleksander Gluszek ${ }^{2}$, Arkadiusz Hudzikowski ${ }^{2}$ and Frank K. Tittel ${ }^{3}$ \\ 1 Institute of Optoelectronics, Military University of Technology, 00-908 Warsaw, Poland \\ 2 Electronics Faculty, Wroclaw University of Science and Technology, 50-370 Wroclaw, Poland; \\ aleksander.gluszek@gmail.com (A.G.); hudzikowski@gmail.com (A.H.) \\ 3 Department of Electrical and Computer Engineering, Rice University, Houston, TX 77005-1892, USA; \\ fkt@rice.edu \\ * Correspondence: jacek.wojtas@wat.edu.pl; Tel.: +48-261-837-943
}

Academic Editors: Paul A. Solomon and Melissa Lunden

Received: 27 January 2017; Accepted: 2 March 2017; Published: 4 March 2017

\begin{abstract}
The application of compact inexpensive trace gas sensor technology to a mid-infrared nitric oxide (NO) detectoion using intracavity quartz-enhanced photoacoustic spectroscopy (I-QEPAS) is reported. A minimum detection limit of $4.8 \mathrm{ppbv}$ within a $30 \mathrm{~ms}$ integration time was demonstrated by using a room-temperature, continuous-wave, distributed-feedback quantum cascade laser (QCL) emitting at $5.263 \mu \mathrm{m}\left(1900.08 \mathrm{~cm}^{-1}\right)$ and a new compact design of a high-finesse bow-tie optical cavity with an integrated resonant quartz tuning fork (QTF). The optimum configuration of the bow-tie cavity was simulated using custom software. Measurements were performed with a wavelength modulation scheme (WM) using a $2 f$ detection procedure.
\end{abstract}

Keywords: laser absorption spectroscopy; bow-tie cavity; I-QEPAS; intracavity quartz-enhanced photoacoustic spectroscopy; mid-infrared trace gas sensors

\section{Introduction}

Optoelectronic gas sensors based on laser absorption spectroscopy (LAS) can achieve low minimum detection limits (MDL) and high selectivity [1,2]. Techniques for improving LAS detection sensitivity are based on multipass gas cells $[3,4]$ or optical resonators (cavities) $[5,6]$ in order to increase the effective optical path length. LAS can be explained by Lambert-Beer's law

$$
I(\lambda)=I_{0}(\lambda) \exp [-\alpha(T, \lambda) L]
$$

where $I_{0}(\lambda)$ is the intensity of the incident radiation, $\alpha$ denotes the absorption coefficient of the targeted trace gas, $L$ is the length of the optical path in the absorbing sample and $T$ is the temperature of the sample [7]. When the wavelength of the incident radiation is matched to an absorption line of the sample, the optical path increases and results in a higher difference between $I_{0}(\lambda)$ and $I(\lambda)$.

An improved MDL can be achieved due to an increase in the signal-to-noise ratio. Wavelength modulation (WM) and frequency modulation (FM) are used to achieve this goal. These methods are applicable for tunable diode lasers absorption spectroscopy (TDLAS-WM/FM) due to the spectral tunability of semiconductor diode lasers. Distributed feedback (DFB) diode lasers are particularly useful for this purpose since they emit a single axial mode output. The wavelength of the probing laser is modulated over the absorption line by a sinusoidal signal of frequency. As a result, the light intensity transmitted through the sample TDLAS gas cell and the signal at the detector has a time-dependent form. The signal is detected with a lock-in amplifier. A slow change of the mean 
laser frequency provides the opportunity to record the first or the second derivative of the absorption spectrum, depending on the demodulation frequency. The advantage of wavelength modulation spectroscopy (WMS) is that it is proportional to the output signal derivative of the targeted absorption line. As a result, harmonic signals reach a maximum value when absorption is the greatest and the odd harmonic signals pass through zero [2]. Therefore, second harmonic (i.e., $2 f$ detection) measurements are usually performed, while the first or the third harmonics are used to stabilize the operating point of the laser source. Hence, good detection sensitivity and selectivity are the main advantages of TDLAS. WM and FM modulation can also be combined with other methods of ultrasensitive laser absorption spectroscopy, such as cavity ring down spectroscopy (CRDS) or photoacoustic spectroscopy (PAS). Trace gas sensor systems based on PAS are very effective tools for trace gas sensing [8-10] and are characterized by a compact, cost-effective and robust architecture. Sensitive microphones are used to detect the conversion of laser light matched to the targeted absorption line in the trace gas sample [11]. The main disadvantage of PAS sensors is their sensitivity to mechanical and acoustic vibrations. A much improved sensor performance can be obtained by replacing the microphone with a commercially available, inexpensive quartz tuning fork (QTF). Such a technique is called quartz-enhanced photoacoustic spectroscopy (QEPAS). QTFs have a resonant frequency of $\sim 32.8 \mathrm{kHz}$ and a Q-factor of $\sim 10^{5}$ in vacuum and $\sim 10^{4}$ at 760 Torr [12]. Furthermore, only the symmetric vibration of a QTF is piezoelectrically active, which makes QEPAS immune to environmental acoustic noise, applicable over a wide range of pressures and capable of analyzing trace gas samples as small as $\sim 3 \mathrm{~mm}^{3}$ [13].

An I-QEPAS sensor platform was first reported by S. Borri et al. based on a combination of QEPAS and an optical resonator [14]. A selective optical filter was used to focus the optical power between the QTF prongs. I-QEPAS achieved an extremely low MDL, because the QEPAS signal amplitude is directly proportional to the available laser excitation power. The target trace gas was carbon dioxide and a $\mathrm{CO}_{2}$ detection sensitivity of $300 \mathrm{ppt}$ for a $4 \mathrm{~s}$ integration time was achieved.

In this paper we report the application of the I-QEPAS sensor platform to fast measurements of nitric oxide trace concentrations. Because of the intracavity power enhancement factor obtained by a four-mirror cavity, improved detection sensitivity was achieved, since in QEPAS the sensitivity scales directly with the laser power. NO is a toxic gas, the concentration of which is strongly related to meteorological conditions and emission sources, which can be both natural and anthropogenic. NO is produced during combustion of fossil fuels in power plants and automobile engines as well as during lightning in thunderstorms. Droplets and vapor of nitric acid are removed from the atmosphere in rain or dry precipitation of aerosols [15]. During daytime, there is a correlation between the concentration of $\mathrm{NO}_{x}$ and $\mathrm{NO}_{2}$, and during nighttime between $\mathrm{NO}_{x}$ and $\mathrm{NO}$. Nitric oxide is also a characteristic decomposition compound of specific explosive materials [7]. Furthermore, NO plays an important role in numerous functions in the human body where it is produced in inflammatory processes. In 1998, three US scientists, Robert F. Furchgott, Louis J. Ignarro and Ferid Murad, received the Nobel Prize in Physiology or Medicine for their discoveries concerning the role of "nitric oxide as a signaling molecule in the cardiovascular system" [16]. Thus, real-time concentration measurements of short-lived NO are important in many applications. At present, most common commercially available NO analyzers, which offer concentration measurements at the ppb level, are based on chemiluminescence. Such analyzers detect the UV radiation produced in the reaction of NO with ozone $[17,18]$. Another approach consists of measuring the change in the ozone concentration instead of the UV intensity [19]. Furthermore, the I-QEPAS-based sensor technology is equally applicable to other trace gas species.

\section{Experimental Setup}

NO has a strong absorption band in the mid-infrared spectral range located at a wavelength of $5.263 \mu \mathrm{m}\left(1900.08 \mathrm{~cm}^{-1}\right)$. The pressure inside the I-QEPAS multipass gas cell (MPGC) was reduced in order to minimize the interference from $\mathrm{CO}_{2}$ and $\mathrm{H}_{2} \mathrm{O}$. The measurements were performed with 
wavelength modulation (WM) using a $2 f$ detection scheme. The WM technique suppresses background noise originating from spectrally non-selective absorbers (such as the resonator walls, QTF electrodes, and MPGC elements). A diagram of the I-QEPAS sensor platform is shown in Figure 1.

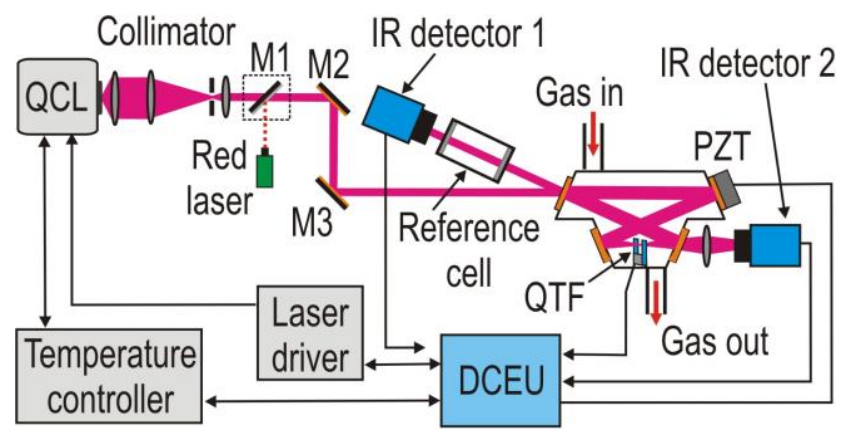

Figure 1. Schematic of an I-QEPAS nitric oxide sensor platform.

A room-temperature, continuous-wave (cw), distributed-feedback (DFB) QCL (Hamamatsu Photonics) emitting at $5.263 \mu \mathrm{m}$ wavelength enclosed in a High Heat Load (HHL) package was used as the excitation source. The linewidth of a DFB QCL is narrow and mainly limited by the noise of the QCL current and temperature controllers [20]. The QCL emission wavelength can be matched to the NO molecular absorption spectrum as shown in Figure 2. Wavelength measurements were carried out using a FT-IR spectrometer (Thermo Fisher Scientific, Waltham, MA, USA). QCL wavelength tuning was demonstrated from $5.255 \mu \mathrm{m}$ (using a laser current of $560 \mathrm{~mA}$ at a laser temperature of $283 \mathrm{~K}$ ) to $5.272 \mu \mathrm{m}$ spectral range (using a laser current of $700 \mathrm{~mA}$ at a laser temperature of $298 \mathrm{~K}$ ).

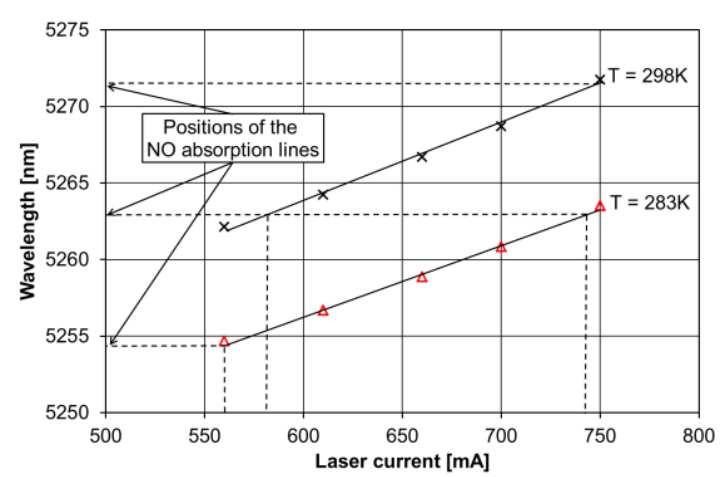

(a)

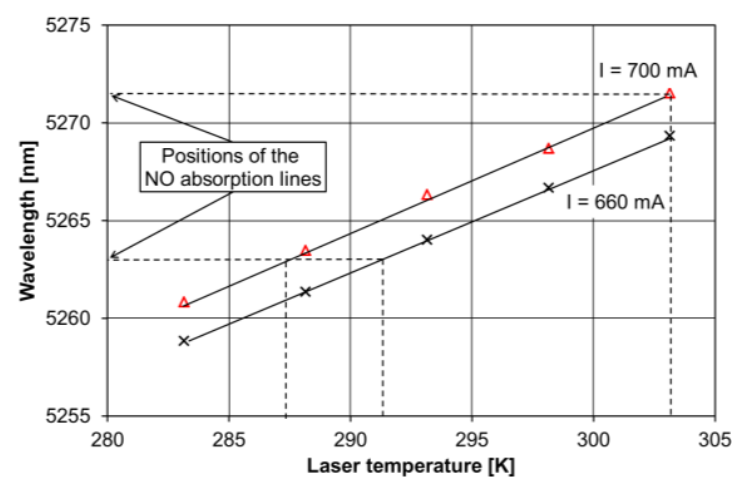

(b)

Figure 2. (a) Measurements results of the QCL current; and (b) the temperature influence on the QCL wavelength.

The QCL radiation was collimated by a $5.95 \mathrm{~mm}$ focal length black diamond aspheric lens (type 390028-E, Thorlabs, Newton, NJ, USA). In addition a collimator consisting two ZnSe lenses and a $300 \mu \mathrm{m}$ pinhole were applied. Such an optical setup resulted in a high power mid-infrared excitation beam (150 mW @ $700 \mathrm{~mA}$ and $283 \mathrm{~K}$ ) with a diameter of $1.7 \mathrm{~mm}$ and a divergence $<1 \mathrm{mrad}$. Figure 3 depicts the available QCL excitation power measured at the collimator output. The QCL power measurements were performed for different QCL currents and temperatures using a power meter (OPHIR-Nova II, Ophir Optronics Solutions Ltd., Jerusalem, Israel). A red diode alignment laser and a movable mirror M1 were used for optimum alignment of the mid-infrared I-QEPAS optical system.

The QCL beam from the collimator was directed by means of two gold coated plane mirrors (M2 and M3) to the compact, high-finesse bow-tie optical cavity. The $12 \mathrm{~cm}^{3}$ bow-tie optical cavity was formed by four half-inch-diameter (two plane and two concave mirrors with a 30 mm radius 
of curvature), highly reflective dielectric mirrors ( $\mathrm{R}>99.99 \%$ from LohnStar Optics, Escondido, CA, USA). The use of such mirrors resulted in a cavity finesse of $7854\left(\mathrm{~F}=\pi / l_{\mathrm{r}}\right.$, where $\mathrm{l}_{\mathrm{r}}$ denotes the losses per round trip due to mirrors transmission). Such an optical resonator design resulted in a power built-up inside the cavity of 2500 times $(\mathrm{G}=\mathrm{F} / \pi)$ [21].

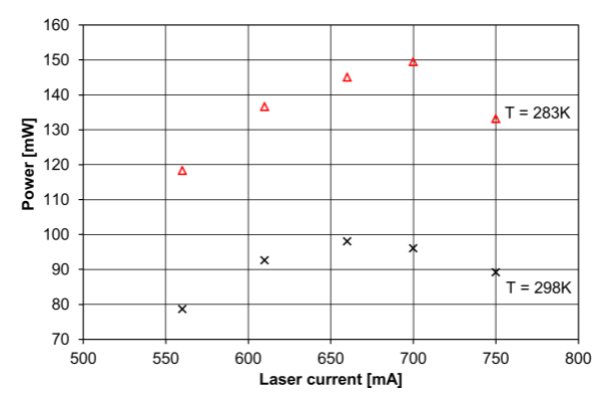

(a)

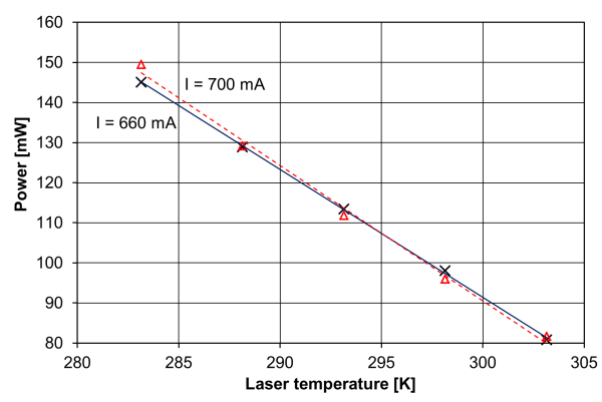

(b)

Figure 3. (a) Measurements results of laser current; and (b) temperature influence on optical power of QCL radiation.

A commercially available quartz tuning fork (QTF) equipped with micro-resonator was applied in our I-QEPAS setup [18]. The QCL beam must pass through the gap between the QTF prongs in order to achieve efficient excitation of the QTF's symmetric vibration. The measurements were performed using a lock-in amplifier and a WM- $2 f$ detection scheme that suppresses the background originating from spectrally non-selective absorbers such as the resonator walls, the QTF electrodes and the gas cell elements. A reference cell filled with a high concentration of the targeted gas and an IR detector-1 were used to register the $1 f$ or $3 f$ signals. Further details related to experimental setup are reported in Section 4.

\section{Cavity Design}

The dimensions and mirror positions of the cavity were specified and designed in Autodesk Inventor software and simulated by means of custom software. The distance between the mirrors was slightly increased ( $0.5 \mathrm{~mm}$ more than the mirror focal point) to achieve a stable, focused beam in the bow-tie cavity based on our simulations. The modeling of the QCL beam reflection on a spherical mirror and the intersection point between a light ray and the mirror sphere was determined first. The intersection points were found with equations reported in Reference [22]. The direction of the reflected beam was calculated based on the law of reflection. Performing a Gaussian laser beam analysis required the generation of a group of ideal rays. Each ray has a different origin of coordinates and different angles (directions) corresponding to the beam size and divergence. Rays are reflected at each mirror $\sim 10^{3}$ times, taking into account associated power losses. Round-off errors were negligible when using 64-bit floating point variables. The QCL beam power at the focal point (waist diameter- $-d_{w}$ ) was determined by the recorded sum of each ray power at the intersection of a plane located at the focal point. Two QCL beam sizes at the focal point were calculated, a first beam size with $50 \%$ of the total power and a second beam size with $99.7 \%(3 \sigma)$ of the total power. Simulations were performed for various QCL beam X-Y positions, divergence angles and beam sizes. The results of the calculation related to cavity performance are presented in Figures 4 and 5 (astigmatic distortions were neglected).

Figure 4 shows that the bow-tie cavity can provide a small waist diameter $(<1 \mathrm{~mm})$ at the QTF position, even for a QCL beam with a $4 \mathrm{~mm}$ diameter, and is stable for varying beam divergences. The bow-tie cavity design is immune to QCL beam displacement. The angles $\theta_{\mathrm{x}}$ and $\theta_{\mathrm{y}}$ should be close to zero in order to achieve the smallest beam waist (Figure 5). For $\theta_{y}$, even a 4 mrad angle leads to a two-times-bigger beam waist diameter for $50 \%$ of the total power. The change of the beam position 
along the $\mathrm{X}$-axis is more important than changes along the $\mathrm{Y}$-axis, where the QCL beam can be moved up to $3 \mathrm{~mm}$ in both directions without significant beam waist changes.

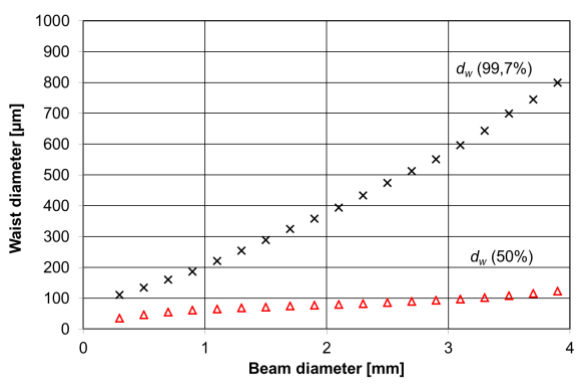

(a)

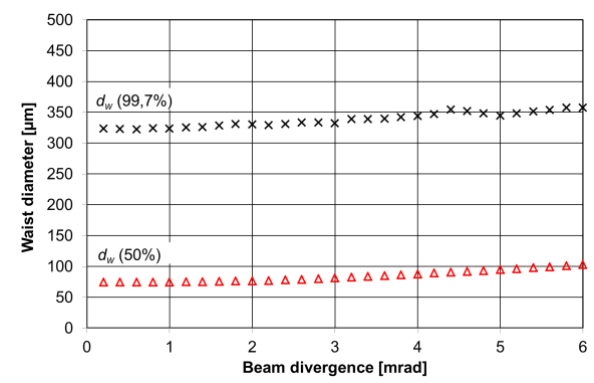

(b)

Figure 4. (a) Simulation results of beam diameter; and (b) divergence of a $4 \mathrm{~mm}$ beam influence on a waist diameter $d_{w}$ inside a four mirror bow-tie cavity.

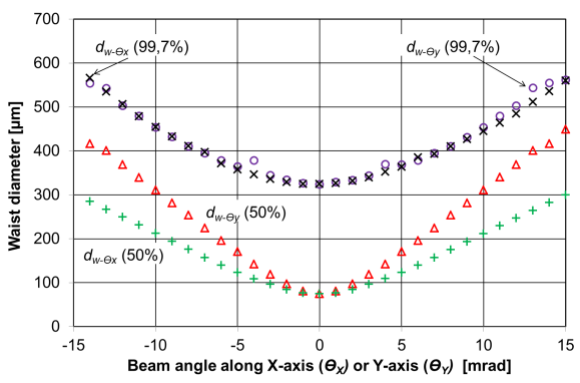

(a)

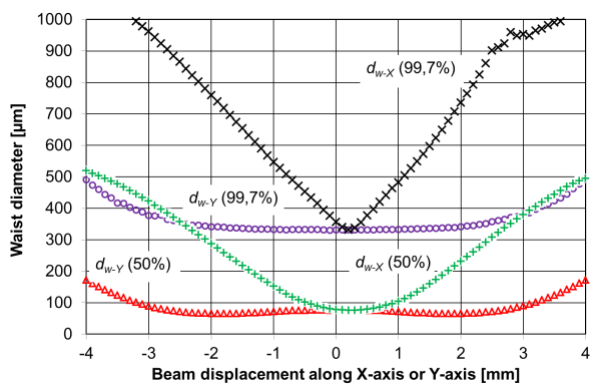

(b)

Figure 5. (a) Results of simulation of the QCL $4 \mathrm{~mm}$ beam waist diameter $\left(d_{w}\right)$ inside the cavity versus the QCL beam angle; and (b) the beam displacement.

\section{Experimental Results}

The bow-tie enclosure was fabricated from an aluminum block with the shape determined by the calculated positions of the mirrors (Figure 6). The angle between the mirrors was $<12^{\circ}$ and the outside dimensions were $68 \times 46 \times 25 \mathrm{~mm}$. The internal and external surfaces of the cavity were the base for all the optical, mechanical and electrical components needed to assemble a complete sensor system consisting of mirrors, the QTF and a piezoelectric transducer (PZT). The mirrors could be aligned precisely by three-point kinematic mounts with metal O-rings and silicone sealing. The QTF could be aligned by means of a sliding mount pressed into the bow-tie enclosure by two springs. This method makes it possible to align the QTF before locking its position by means of epoxy.

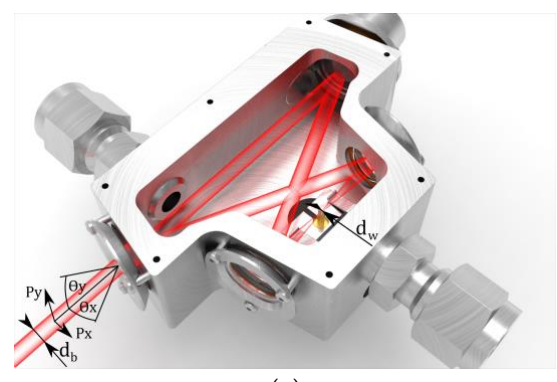

(a)

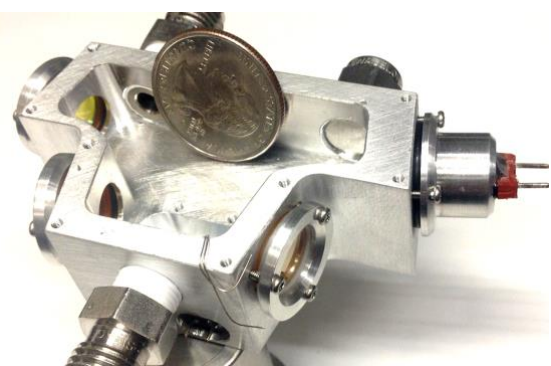

(b)

Figure 6. (a) Optical cavity 3D design; and (b) photo of a novel and compact bow-tie resonator for an I-QEPAS sensor system. 
Interference from the cavity mirror feedback can be eliminated, because the QCL beam can be injected at a small angle with respect to the input mirror axis. Hence, a costly optical isolator was not required. In the beam reflected by the input mirror, a reference gas cell filled with pure $\mathrm{NO}(3 \mathrm{~cm}$ in length and a $0.85 \mathrm{~cm}$ diameter) and a mercury cadmium-telluride (MCT) detector-1 (PVI-4TE, VIGO System S.A., Ozarow Mazowiecki, Poland) were placed for QCL wavelength monitoring.

The total cavity length was $L=193 \mathrm{~mm}$, corresponding to a free spectral range (FSR $=\mathrm{c} / \mathrm{L}$ ) of $1.55 \mathrm{GHz}$. One of the plane mirrors was mounted on a PZT and provided precise tuning of the cavity length in the range of $11 \mu \mathrm{m}$ along the z-axis to achieve mode matching with the QCL radiation. The intracavity optical power was determined to be $\sim 170 \mathrm{~W}$ by measuring the optical power reflected from the input cavity mirror. Hence, a power enhancement factor of 1276 was achieved with respect to the incident QCL power of $133.2 \mathrm{~mW}$ achieved with a QCL drive current of $742.5 \mathrm{~mA}$ and an operating temperature of $283 \mathrm{~K}$.

The cavity was designed to focus the laser beam between the two spherical mirrors. At the focal plane of these two mirrors, the QTF was mounted on a custom-machined miniature xyz translator. The QCL beam passed through a $0.5 \mathrm{~mm}$ gap between the QTF prongs without hitting them. The electrical parameters of the QTF (i.e., the dynamical resistance, the quality factor $Q$ and the resonant frequency $f_{0}$ ) were measured with a function generator (DS345, Stanford Research System), a lock-in amplifier (SRS830, Stanford Research System) and LabVIEW software. The measured QTF resonance frequency was $f_{0}=32,776.2 \mathrm{~Hz}$ and the quality factor $Q$ exceeded 28,900 (see Figure 7).

The experimental setup is depicted in Figure 8. A MCT detection module (PVI-4TE, VIGO System S.A.) detector-2 was used to measure the exiting QCL radiation. A custom digital control electronic unit (DCEU) based on a STM32F7 microcontroller platform equipped with an analog front-end system was developed to generate all the signals required by the I-QEPAS sensor system. The modulation of the QCL current was performed by a low-amplitude sinusoidal signal with a frequency corresponding to half of the QTF resonance frequency, $f_{0} / 2(\sim 16.4 \mathrm{kHz})$. The output signal from the QTF was connected to a transimpedance preamplifier and subsequently directed to a custom lock-in amplifier detecting the second harmonic of the QTF signal $(\sim 32.8 \mathrm{kHz})$.

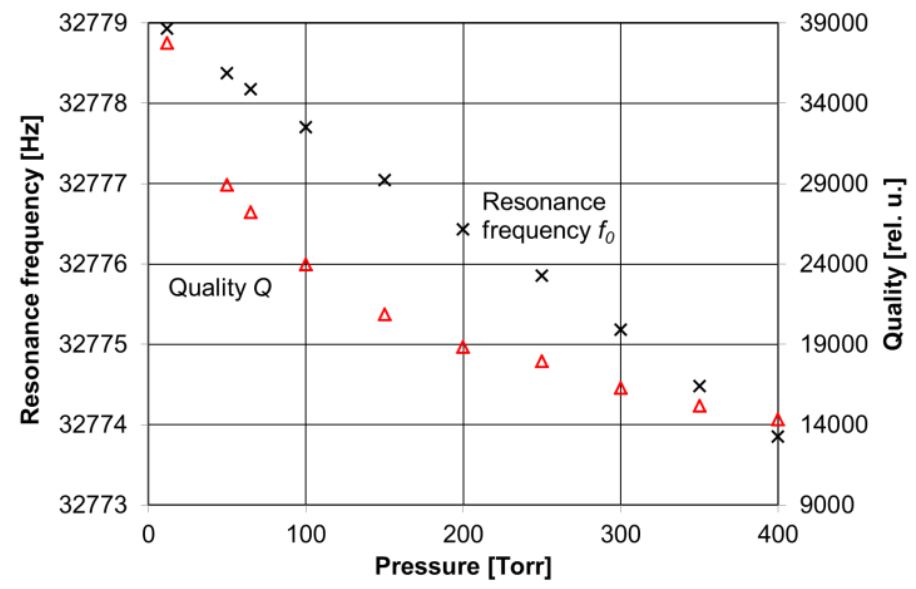

Figure 7. QTF resonance frequency and quality factor measurements as a function of pressure inside the optical cavity.

The PZT transducer shown in Figures 1 and 6 was controlled by a digital high-speed feedback loop using the signal from the IR detector-2. This signal tracked the amplitude of the I-QEPAS signal in order to achieve the highest power inside the four-mirror optical resonator. The PZT driving signal and output detector- 2 signal are shown in Figure $8 \mathrm{~b}$. Thin lines registered by detector- 2 are the result of the high finesse of the cavity. 


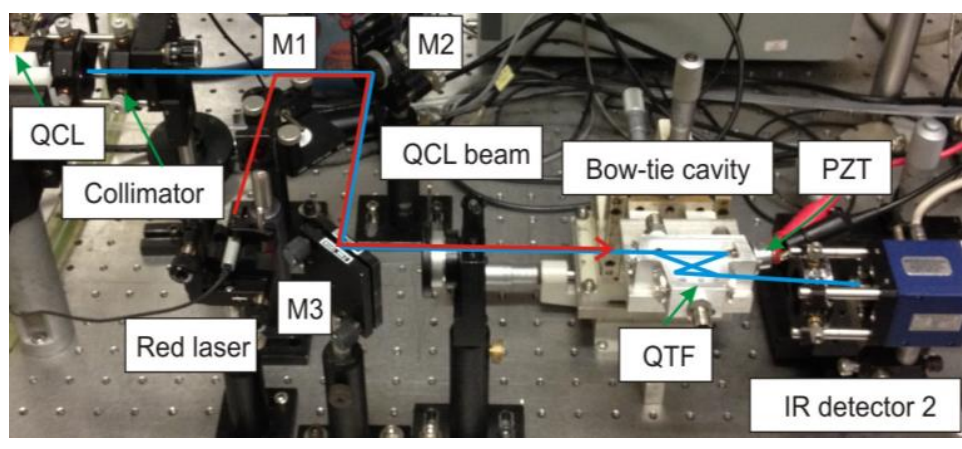

(a)

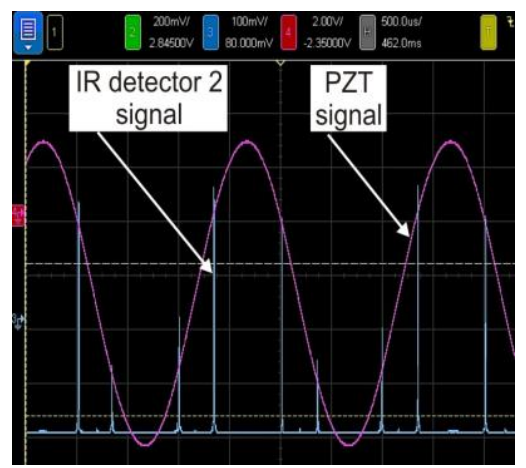

(b)

Figure 8. (a) Photos of the experimental setup; and (b) an example of recorded PZT and I-QEPAS signals.

The I-QEPAS-based NO concentration measurements were carried out in a locked mode. The QCL frequency was initially set to the center of the absorption line and the signal strength was monitored by the IR detector-2. A custom algorithm based on a PID (Proportional, Integral, Derivative algorithm) with conditional executing and phase wrapping functions provided the signal to control the PZT driver. Such a feedback loop maintained a high QCL power inside the bow-tie cavity.

The absorption line at $1900.08 \mathrm{~cm}^{-1}$ was found to be optimal for NO detection, as already mentioned in Section 2. An NO absorption cross-section of $0.7 \times 10^{-18} \mathrm{~cm}^{2}$ was observed at this wavenumber. A detailed description of $\mathrm{NO}$ absorption line selection can be found in Reference [23]. Measurements of a 1 ppm NO sample at 50 Torr were carried out. Both the gas pressure and the wavelength modulation depth to optimize the NO I-QEPAS detection performance were determined experimentally. The optimal amplitude of the modulation signal for scanning of the cavity mode (FWHM $\sim 0.2 \mathrm{MHz}$ ) was obtained by measuring the I-QEPAS signal as a function of the QCL modulation current at the optimum pressure inside the MPGC. A critical task was to minimize the noise of the QCL controller using low-noise custom electronics because of the narrow cavity mode FWHM. To determine the MDL, defined as the lowest amount of trace gas that can be distinguished from the system noise, we used a signal-to-noise ratio (SNR) and a 90\% confidence level that corresponds to a 1.645 SD (standard deviation). Figure 9 shows the NO concentration measurements results. The sensor signal was measured with a $1.01 \mathrm{ppm} \mathrm{NO}$ of a high-purity calibration mixture and the noise as fluctuations of this concentration level. The measurements were carried out with a $30 \mathrm{~ms}$ integration time. A difference smaller than $0.5 \%$ between the average value of the measurements and the nominal concentration of the calibration mixture was measured.

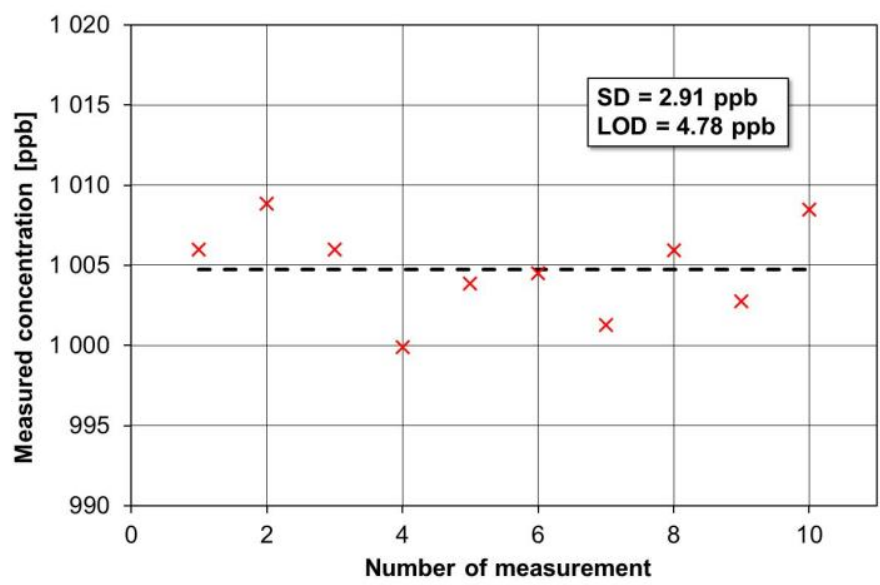

Figure 9. Results of NO concentration measurements. 
The MDL of the I-QEPAS NO sensor operating in a locked mode at $\lambda \sim 5.263 \mu \mathrm{m}$ was $\sim 4.8 \mathrm{ppbv}$ with a $30 \mathrm{~ms}$ integration time due to the cavity stability and effective locking between the QCL and the bow-tie cavity. The MDL was limited by distortions of the PZT driving signal and its adverse influence on the NO concentration measurements. This effect occurred because the PZT required a high-amplitude voltage signal (up to $10 \mathrm{~V}$ ) and its harmonics were located near the output signal frequency.

\section{Conclusions}

In conclusion, the first demonstration of intracavity quartz-enhanced photoacoustic spectroscopy applied to mid-infrared NO detection using a compact bow-tie cavity configuration with a minimum detection limit of $\sim 4.8 \mathrm{ppbv}$ was reported. The bow-tie cavity provided a high finesse and a small waist diameter $(<1 \mathrm{~mm})$ as well as a minimum beam divergence and beam displacement. Low-noise digital electronics were developed to ensure the effective coupling of the CW, DFB QCL emission line to a narrow bow-tie cavity mode. Further improvements of minimizing the influence of the PZT noise can improve the MDL by approximately one order of magnitude.

An I-QEPAS-based sensor is a novel ultra-sensitive and selective platform for trace gas sensing and is useful in many applications including environmental monitoring, industrial emission measurements, chemical analysis, security and medical diagnostics [24] as well as in the life sciences $[1,25]$. NO plays a very important role in atmospheric chemistry as it controls the ozone concentration and production rates in the stratosphere by the photodissociation process [15]. Other trace gases detectable by I-QEPAS include $\mathrm{NO}_{2}, \mathrm{~N}_{2} \mathrm{O}, \mathrm{HNO}_{3}, \mathrm{CH}_{2} \mathrm{O}, \mathrm{SO}_{2}, \mathrm{O}_{3}, \mathrm{CO}$ and $\mathrm{NH}_{3}$. Furthermore, I-QEPAS sensors are ultra-sensitive, compact and less costly than techniques that require costly photodetectors.

Acknowledgments: J. Wojtas acknowledges financial support by the Polish National Center for Research and Development (Research project ID: 179616). F.K. Tittel acknowledges support by the National Science Foundation (NSF) ERC MIRTHE award and the Robert Welch Foundation (Grant C-0586).

Author Contributions: J.W. and F.K.T. conceived and designed the experiments; A.H. performed the cavity simulations, developed the custom digital control electronic unit and provided their descriptions; A.G. designed the cavity, implemented it in the setup and made the corresponding descriptions. All authors were involved in the setup alignment and NO concentration measurements; J.W. performed QCL and QTF parameter measurements, analyzed the research data and wrote the paper based on the input from the other authors.

Conflicts of Interest: The authors declare no conflict of interest.

\section{References}

1. Lewicki, R.; Doty, J.H.; Curl, R.F.; Tittel, F.K.; Wysocki, G. Ultrasensitive detection of nitric oxide at $5.33 \mu \mathrm{m}$ by using external cavity quantum cascade laser-based Faraday rotation spectroscopy. Proc. Natl. Acad. Sci. USA 2009, 106, 12587-12592. [CrossRef] [PubMed]

2. Liu, J.T.C.; Jeffries, J.B.; Hanson, R.K. Wavelength modulation absorption spectroscopy with $2 f$ detection using multiplexed diode lasers for rapid temperature measurements in gaseous flows. Appl. Phys. B 2004, 78, 503-511. [CrossRef]

3. Jean-Franqois, D.; Ritz, D.; Carlier, P. Multiple-pass cell for very-long-path infrared spectrometry. Appl. Opt. 1999, 38, 4145-4150.

4. Nelson, D.; Shorter, J.; McManus, J.; Zahniser, M.S. Sub-part-per-billion detection of nitric oxide in air using a thermoelectrically cooled mid-infrared quantum cascade laser spectrometer. Appl. Phys. B 2002, 75, 343-350. [CrossRef]

5. Kosterev, A.A.; Malinovsky, A.L.; Tittel, F.K.; Gmachl, C.; Capasso, F.; Sivco, D.L.; Baillargeon, J.N.; Hutchinson, A.L.; Cho, A.Y. Cavity ringdown spectroscopic detection of nitric oxide with a continuous-wave quantum-cascade laser. Appl. Opt. 2001, 40, 5522-5529. [CrossRef] [PubMed]

6. Berden, G.; Peeters, R.; Meijer, G. Cavity ring-down spectroscopy: Experimental schemes and applications. Int. Rev. Phys. Chem. 2000, 4, 565-607. [CrossRef] 
7. Wojtas, J.; Stacewicz, T.; Bielecki, Z.; Rutecka, B.; Medrzycki, R.; Mikolajczyk, J. Towards optoelectronic detection of explosives. Opt. Electron. Rev. 2013, 21, 210-219. [CrossRef]

8. Kachanov, A.; Koulikov, S.; Tittel, F.K. Cavity Enhanced Optical Feedback Assisted Photoacoustic Spectroscopy with a 10.4 microns External-Cavity Quantum Cascade Laser. Appl. Phys. B 2013, 110, 47-56. [CrossRef]

9. Zeninari, V.; Kapitanov, V.A.; Courtois, D.; Ponomarev, Y.N. Design and characteristics of a differential Helmholtz resonant photoacoustic cell for infrared gas detection. Infrared Phys. Technol. 1999, 40, 1-23. [CrossRef]

10. Miklos, A.; Hess, P.; Bozoki, Z. Application of acoustic resonators in photoacoustic trace gas analysis and metrology. Rev. Sci. Instrum. 2001, 72, 1937-1955. [CrossRef]

11. Bozoki, Z.; Mohacsi, A.; Szabo, G.; Bor, Z.; Erdelyi, M.; Chen, W.; Tittel, F.K. Near Infrared Diode Based Spectroscopic Detection of Ammonia: A Comparative Study of Photoacoustic and Direct Optical Absorption Methods. Appl. Spectrosc. 2002, 56, 715-719. [CrossRef] [PubMed]

12. Curl, R.F.; Capasso, F.; Gmachl, C.; Kosterev, A.A.; McManus, B.; Lewicki, R.; Pusharsky, M.; Wysocki, G.; Tittel, F.K. Quantum cascade lasers in chemical physics. Chem. Phys. Lett. 2010, 487, 1-18. [CrossRef]

13. Kosterev, A.A.; Bakhirkin, Y.A.; Curl, R.F.; Tittel, F.K. Quartz-enhanced photoacoustic spectroscopy. Opt. Lett. 2002, 27, 1902-1904. [CrossRef] [PubMed]

14. Borri, S.; Patimisco, P.; Galli, I.; Mazzotti, D.; Giusfredi, G.; Akikusa, N.; Yamanishi, M.; Scamarcio, G.; De Natale, P.; Spagnolo, V. Intracavity quartz-enhanced photoacoustic sensor. Appl. Phys. Lett. 2014, 104, 091114. [CrossRef]

15. Portmann, R.W.; Daniel, J.S.; Ravishankara, A.R. Stratospheric ozone depletion due to nitrous oxide: influences of other gases. Phil. Trans. R. Soc. B 2012, 367, 1256-1264. [CrossRef] [PubMed]

16. The Nobel Prize in Physiology or Medicine 1998. Available online: http://www.nobelprize.org/nobel_ prizes/medicine/laureates/1998/press.html (accessed on 15 November 2016).

17. Sievers Nitric Oxide Analyzer Overview. Available online: http://www.geinstruments.com/products-andservices/nitric-oxide-analyzer (accessed on 17 February 2017).

18. Chemiluminescence NO/NOx Gas Analysis. Available online: http://www.k2bw.com/5_c_31.htm (accessed on 17 February 2017).

19. Model 410 Nitric Oxide Monitor. Available online: http://www.twobtech.com/model-410-no-monitor.html (accessed on 17 February 2017).

20. Tombez, L.; Schilt, S.; di Francesco, J.; di Domenico, G.; Hofstetter, D.; Thomann, P. Linewidth of a quantum-cascade laser assessed from its frequency noise spectrum and impact of the current driver. Appl. Phys. B Lasers Opt. 2012, 109, 407-414. [CrossRef]

21. Saleh, B.E.A.; Teich, M.C. Fundamentals of Photonics; John Wiley \& Sons Inc.: New York, NY, USA, 1991; pp. 310-340.

22. Glassner, A. Graphics Gems; Academic Press: New York, NY, USA, 1990; pp. 388-389.

23. Wojtas, J.; Mikolajczyk, J.; Bielecki, Z. Aspects of the Application of Cavity Enhanced Spectroscopy to Nitrogen Oxides Detection. Sensors 2013, 13, 7570-7598. [CrossRef] [PubMed]

24. Patimisco, P.; Borri, S.; Galli, I.; Mazzotti, D.; Giusfredi, G.; Akikusa, N.; Yamanishi, M.; Scamarcio, G.; De Natale, P.; Spagnolo, V. High finesse optical cavity coupled with a quartz enhanced photoacoustic spectroscopic sensor. Analyst 2015, 140, 736-743. [CrossRef] [PubMed]

25. Buszewski, B.; Grzywinski, D.; Ligor, T.; Stacewicz, T.; Bielecki, Z.; Wojtas, J. Detection of volatile organic compounds as biomarkers in breath analysis by different analytical techniques. Bioanalysis 2013, 5, 2287-2306. [CrossRef] [PubMed]

(C) 2017 by the authors. Licensee MDPI, Basel, Switzerland. This article is an open access article distributed under the terms and conditions of the Creative Commons Attribution (CC BY) license (http:/ / creativecommons.org/licenses/by/4.0/). 